\title{
Suggestive association between variants in IL1RAPL and asthma symptoms in Latin American children
}

\author{
Cintia Rodrigues Marques ${ }^{1}$, Gustavo NO Costa ${ }^{2}$, Thiago Magalhães da Silva ${ }^{2,3}$, Pablo Oliveira ${ }^{2,4}$, \\ Alvaro A Cruz ${ }^{5}$, Neuza Maria Alcantara-Neves ${ }^{1}$, Rosemeire L Fiaccone ${ }^{6}$, Bernardo L Horta ${ }^{7}$, \\ Fernando Pires Hartwig ${ }^{7}$, Esteban G Burchard ${ }^{8,9}$, Maria Pino-Yanes ${ }^{10,11}$, Laura C Rodrigues ${ }^{12}$, \\ Maria Fernanda Lima-Costa ${ }^{13}$, Alexandre C Pereira ${ }^{14}$, Mateus H Gouveia ${ }^{15}$, Hanaisa P Sant Anna ${ }^{15}$, \\ Eduardo Tarazona-Santos ${ }^{15}$, Maurício Lima Barreto ${ }^{2,4}$ and Camila Alexandrina Figueiredo $o^{\star, 1}$
}

\begin{abstract}
Several genome-wide association studies have been conducted to investigate the influence of genetic polymorphisms in the development of allergic diseases, but few of them have included the $X$ chromosome. The aim of present study was to perform an X chromosome-wide association study (X-WAS) for asthma symptoms. The study included 1307 children of which 294 were asthma cases. DNA was genotyped using 2.5 HumanOmni Beadchip from Illumina. Statistical analyses were performed in PLINK 1.9, MACH 1.0 and Minimac2. The variant rs12007907 (g.29483892C > A) in IL1RAPL gene was suggestively associated with asthma symptoms in discovery set (odds ratio $(\mathrm{OR})=0.49,95 \%$ confidence interval $(\mathrm{CI}): 0.37-0.67 ; P=3.33 \times 10^{-6}$ ). This result was replicated in the ProAr cohort in men only $(\mathrm{OR}=0.45,95 \% \mathrm{Cl}: 0.21-0.95 ; P=0.038)$. Furthermore, investigating the functional role of the rs12007907 on the production a Th2-type cytokine, IL-13, we found a negative association between the minor allele A with IL-13 production in the discovery set $(P=0.044)$. Gene-based analysis revealed that NUDT10 was the most consistently associated with asthma symptoms in discovery sample. In conclusion, the rs 12007907 variant in IL1RAPL gene was negatively associated with asthma and IL-13 production in our study and a sex-specific association was observed in one of the validation samples. It suggests an effect on asthma susceptibility and may explain differences in severe asthma frequency between women and men.
\end{abstract}

European Journal of Human Genetics (2017) 25, 439-445; doi:10.1038/ejhg.2016.197; published online 25 January 2017

\section{INTRODUCTION}

Asthma affects the low airways resulting in airflow obstruction, bronchial hyper-responsiveness and inflammation. ${ }^{1}$ The most recent Global Asthma Report estimates that over 334 million people are affected by asthma worldwide. ${ }^{2}$ This prevalence is increasing in recent decades among individuals living in industrialized countries and, more recently, in developing countries. Therefore, asthma is considered a public health problem. ${ }^{3-6}$ Asthma is a complex disease and the mechanisms responsible for its causation are not fully understood, although it is clear that genetic and environment factors play an important role in its pathogenesis. ${ }^{7,8}$

Many genome-wide association studies (GWAS) have been conducted to investigate the influence of genetic polymorphisms in the development of several diseases. It is estimated that almost 1400 GWAS have identified $\sim 2800$ peak associations $\left(P \leq 5.10^{-8}\right)$ so far. ${ }^{9}$ However, $<30 \%$ of these studies report the $\mathrm{X}$ chromosome in their analysis. This may happened for several reasons, including the limited number of X chromosome single-nucleotide polymorphisms (SNPs) presented in some genotyping platforms and the difference in the number of $\mathrm{X}$ chromosome copies between men and women, which complicate the association analysis.

The X chromosome contains 2300 genes ${ }^{10}$ and almost $1,3 \mathrm{M}$ SNPs. ${ }^{4}$ Genes present in the $\mathrm{X}$ chromosome are associated with different genetic diseases such as Duchenne muscular dystrophy, ${ }^{11}$ Hemophylia $\mathrm{A}^{12}$ and IPEX (immune dysfunction, polyendocrinopathy, enteropathy and X-linked) syndrome. ${ }^{13}$ In addition, this chromosome includes genes that have been associated with allergic diseases, such as FOXP $3^{14}$ and others, which may be of relevance in the context of asthma, such as the TLR7 and TLR8. ${ }^{15}$ Thus, analyzing SNPs present in this chromosome may provide important insights regarding genetic variants associated with diseases and should not be neglected. This is of special interest in the asthma context, where differences in prevalence, morbidity and severity of the disease according to gender have been reported. ${ }^{16,17}$ These studies have shown that males are more affected during early childhood but a gender shift has been observed at puberty, with a higher prevalence of asthma in females at adulthood.

\footnotetext{
${ }^{1}$ Instituto de Ciências da Saúde, Universidade Federal da Bahia, Salvador, Brazil; ${ }^{2}$ Instituto de Saúde Coletiva, Universidade Federal da Bahia, Salvador, Brazil; ${ }^{3}$ Departamento de Ciências Biológicas, Universidade Estadual do Sudoeste da Bahia, Jequié, Brazil; ${ }^{4}$ Centro de Pesquisas Gonçalo Muniz, FIOCRUZ, Salvador, Brazil; ${ }^{5}$ School of Medicine, ProARCenter of Excellence in Asthma, Federal University of Bahia School of Medicine, Salvador, Brazil; ${ }^{6}$ Departamento de Estatística, Instituto de Matemática, Universidade Federal da Bahia, Salvador, Brazil; ${ }^{7}$ Departamento de Medicina Social, Programa de Pós-Graduação em Epidemiologia, Universidade Federal de Pelotas, Pelotas, Brazil; ${ }^{8}$ Department of Medicine, University of California, San Francisco, CA, USA; ${ }^{9}$ Department of Bioengineering and Therapeutic Sciences, University of California, San Francisco, CA, USA; ${ }^{10}$ Department of Medicine, Hospital Universitario N.S. de Candelaria, Tenerife, Spain; ${ }^{11}$ Department of Medicine, University of California, San Francisco (UCSF), CA, USA; ${ }^{12}$ Department of Infectious Disease Epidemiology, Faculty of Epidemiology, London School of Hygiene and Tropical Medicine; ${ }^{13}$ Instituto de Pesquisa Rene Rachou, Fundação Oswaldo Cruz. Av. Augusto de Lima, Belo Horizonte, Minas Gerais, Brazil; ${ }^{14}$ Faculdade de Medicina, Instituto do Coração, Universidade de São Paulo, São Paulo, Brazil; ${ }^{15}$ Departamento de Biologia Geral, Instituto de Ciências Biológicas, Universidade Federal de Minas Gerais, Belo Horizonte, Minas Gerais, Brazil

*Correspondence: Professor CA Figueiredo, Instituto de Ciências da Saúde, Universidade Federal da Bahia, Avenida Reitor Miguel Calmon, s/ no, Canela, Salvador CEP_-40110100, Brazil. Fax: +55 71 32838921; E-mail: cavfigueiredo@gmail.com

Received 2 May 2016; revised 22 November 2016; accepted 6 December 2016; published online 25 January 2017
} 
Factors contributing to such sex difference remain unclear and may involve hormonal changes together with genetic predisposition.

Considering the presence of important genes on the $\mathrm{X}$ chromosome, possible associations with complex diseases and difficulties in analyzing X-linked markers in GWAS, several studies have analyzed the X chromosome separately. ${ }^{18-20}$ Therefore, the aim of this study was to perform an $\mathrm{X}$ chromosome-wide association study (X-WAS) for asthma symptoms in childhood. To our knowledge, this is the first $\mathrm{X}$-WAS for asthma conducted worldwide.

\section{METHODS}

\section{Study design and population characterization}

As described in previous publications, ${ }^{21-23}$ Social Changes, Asthma and Allergy in Latin America (SCAALA) is a research program conducted in Brazil and Ecuador. The Brazilian component comprised a cohort of 1307 (705 males) unrelated children between 4 and 11 years old. Asthma symptoms were defined and classified as reported in a previous publication of SCAALA Salvador studies. ${ }^{24}$ Briefly, children were classified as having current wheeze as previously described ${ }^{25,26}$ by using phase II International Study of Asthma and Allergies in Childhood questionnaire (wheezing in the last 12 months) and were considered to have current wheeze plus symptoms if parents reported wheezing in the previous 12 months and at least one of the following: (1) diagnosis of asthma ever; (2) wheezing with exercise in the last 12 months; (3) four or more episodes of wheezing in the last 12 months; and (4) waking up at night because of wheezing in the last 12 months. Ethical approval for this study was obtained from the Brazilian National Ethical Council, and written informed consent was obtained from the guardian of each child.

\section{Genotyping and quality control}

DNA was extracted from peripheral blood using a commercial kit (Gentra Puregene Blood Kit (Qiagen, Germantown, ML, USA)). Subjects were genotyped using the 2.5HumanOmni Beadchip from Illumina (San Diego, CA, USA). There were 46945 SNPs genotyped on X chromosome and 391 SNPs in pseudoautossomal regions between the $\mathrm{X}$ and $\mathrm{Y}$ chromosomes. Genotyping quality control and data cleaning was performed as specified in Kehdy et al. ${ }^{27}$ Closely related individuals $(n=64)$ identified as in Kehdy et al. ${ }^{27}$ were excluded. SNPs were removed if MAF (minor allele frequency) $\leq 1 \%$ in males and females separately. SNPs were also excluded if MIF (missing frequency) was $\geq 2 \%$ threshold either the separated missing frequencies in males and females (MIF males-MIF females). Genotyping data has been deposited at the European Genome-phenome Archive (EGA, http://www.ebi.ac. uk.ega/), which is hosted by the EBI, under accession number EGAS00001001245.

\section{Sequence annotations}

Comparative genomic data and regulatory features for the IL1RAPL1 gene (X: 29418224-29545784; GRCh37/hg19 reference sequence) were obtained from both the Ensembl (http://www.ensembl.org) and University of California Santa Cruz (http://genome.ucsc.edu) genome browsers. SNP positions were cross-referenced with sequence annotations, including genomic evolutionary rate profiling-constrained elements for 36 eutherian mammals (GERP-EPO low coverage), ${ }^{28}$ chromatin segmentation state, and enrichment for marks of open chromatin (DNase I hypersensitive sites). These last two types of information were obtained from the ENCODE project. ${ }^{29}$

\section{Validation studies}

Validation analyses were performed in independent Brazil and US Latin American populations. The US Latin American samples dates were collected from Genes-environments and Admixture in Latino Americans study (GALA II). The GALA II study is an ongoing multicenter case-control study of asthma in Latino children and adolescents (1893 asthma cases and 1881 controls), organized from the coordinating center based at the University of California, San Francisco, CA, USA. ${ }^{30}$ Subjects were eligible if they were 8-21 years old and had no history of other lung or other chronic illnesses. Asthma was defined based on physician diagnosis and report of symptoms and medication use within the past 2 years before the recruitment. Controls had no reported history of asthma, lung disease or chronic illness, allergic disease (eczema, hives, hay fever and allergic rhinitis), no reported use of medication for allergies, and no reported symptoms of coughing, wheezing or shortness of breath in their lifetime.

The second replication set was the Pelotas birth cohort from Brazil. ${ }^{31}$ It is comprised of 1151 participants (343 asthma cases and 808 health individuals), which were interviewed when they were 18 years of age. Asthma was defined based on the same criteria used to case definition in the SCAALA cohort.

The third replication set was the Asthma and Allergic Rhinitis Control Program in Bahia (ProAR). Cases were comprised of 420 patients with severe asthma and 305 patients with mild or intermittent control persistent asthma according to the Global Initiative rating against Asthma $^{32}$ from both genders and age $\geq 18$ years, living in Salvador. Controls were comprised with 398 subjects without history of asthma, residing in Salvador and unrelated of the cases. In this replication study, the rs 12007907 genotyping was performed using TaqMan probe-based 5'-nuclease assays (Applied Biosystems, Foster City, CA, USA) in QuantStudio 12k Flex equipment.

\section{Cytokine production}

Venous blood was collected into heparinised tubes and the collected cells were cultured as previously described by Figueiredo et al. ${ }^{33}$ The production IL-5 and IL-13 upon pokeweed (PWM) stimulation was measured in whole-blood culture supernatants using commercially available antibody pairs and recombinant cytokine standards (BD Biosciences Pharmingen, San Diego, CA, USA) by sandwich ELISA according to the manufacturer's instructions. Cytokine concentrations were determined by interpolation of standard curves. ${ }^{33}$

\section{Statistical analysis}

Logistic regression analyses using additive genetic model for asthma were performed in PLINK 1.9 after filters application for quality control procedures. Analyses were adjusted by sex, age and the first three principal components (PCs) delineated through Eigenstrat on 370539 genome-wide autosomal SNPs as covariates. Because of sex-biased pattern of admixture in Brazilian populations, with preferential mating between males with predominant European ancestry and women with predominant African or Amerindian ancestry, ${ }^{34}$ the non-European ancestry inferred to the $\mathrm{X}$ chromosome tend to be higher than estimated for the autosomes. ${ }^{27}$ For this reason, the average local ancestry inferred for X chromosome has been used as covariate in some analyzes. Details about the methodology used to infer X chromosome ancestry are described elsewhere. ${ }^{27}$ Based on the default of PLINK, the analysis were performed assuming no dosage compensation. Thus male genotypes were coded as 0 or 1 and female genotypes were coded as 0,1 or 2. Sex-stratified analyses were performed for taking into account the X-inactivation and different effect sizes of X-linked SNPs between males and females. For this, data from each sex (cases and controls) were analyzed separately and then combined using a weighted Stouffer's method. ${ }^{35}$ To test for differences in effect size according to sex we performed tests of heterogeneity using the METAL program. ${ }^{35}$ The SNP imputation pre-phase for the first step was performed in $\mathrm{MACH}$ 1.0 and the second phase in Minimac2 $2^{36,37}$ and was run separately for males and females. For imputation we used as reference the African population (1000G.AFR, 20100804.tgz) according 1000 genomes. ${ }^{4}$ We imputed with high confidence $\left(R^{2}=0.3\right)$. To obtain a normal distribution of cytokines values a $\log$ transformation was applied and the differences in cytokines concentration among the rs12007907 genotypes were analyzed using linear regression adjusted for sex, age and the three PCs of genetic ancestry using 370539 genome-wide autosomal SNPs.

\section{Gene-based association analysis}

Gene-based association tests were performed through VEGAS2 $2^{38}$ and MAGMA ${ }^{39}$ software. Briefly, this approach aggregate association signals considering all SNPs within a gene while account for dependence between signals due to linkage disequilibrium (LD) to assign a level of significance for the association of the gene overall. In addition, MAGMA also performs gene-based analysis using PC regression and this method was applied for comparison with the results based on $P$-value that were obtained in VEGAS2 and MAGMA. In all gene-based analyzes 
empirical $P$-values were obtained through permutational methods, being performed 10.000 simulations in VEGAS2 and 100.000 in MAGMA.

\section{RESULTS}

The final data set, after quality control for SNPs and individuals, included 35410 SNPs and 1246 samples (280 cases). We have identified two markers in X chromosome suggestively associated with asthma symptoms (Figure 1). Table 1 presents the top 20 SNPs ranked according to $P$-value. The strongest association was observed for the SNP rs12007907 (odds ratio $(\mathrm{OR})=0.49,95 \%$ confidence interval (CI): $\left.0.37-0.67 ; P=3.33 \times 10^{-6}\right)$, which is an intronic variant located in the ILIRAPL1 (IL-1 R acessory protein-like) gene. The second most significantly associated variant was the $\mathrm{rs} 199522937(\mathrm{OR}=1.71$, 95\% CI: $1.35-2.14 ; P=5.11 \times 10^{-6}$ ) located in an intergenic region

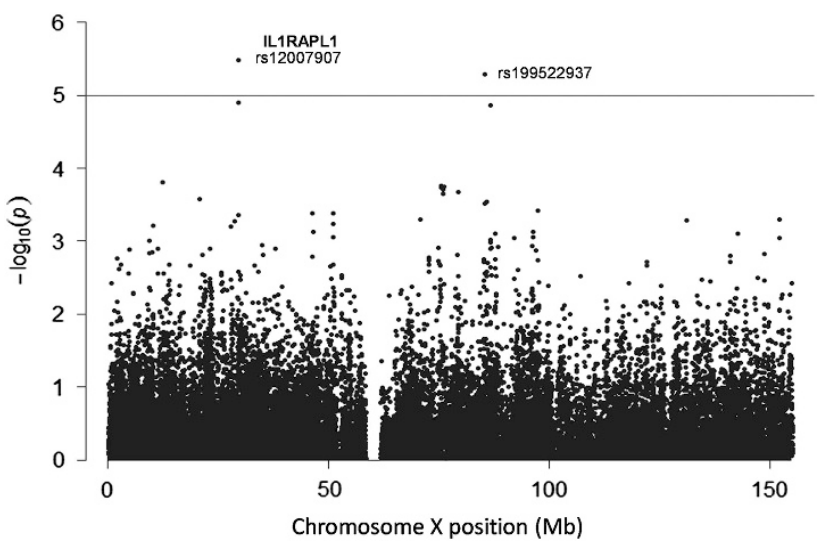

Figure 1 Manhattan plot of $X$ chromosome-wide association results. The line corresponds to threshold for suggestive associations $\left(P<5.0 \times 10^{-6}\right)$ adopted in logistic regression analyses performed using additive genetic model for asthma and adjusted by sex, age and the first three principal components of ancestry markers.
$5 \mathrm{~kb}$ upstream of the $\mathrm{DACH} 2$ gene (Dachshund Family Transcription Factor). These results were virtually unchanged when using the ancestry inferred to the $\mathrm{X}$ chromosome to adjust by population stratification $\left(\mathrm{OR}=0.49,95 \% \mathrm{CI}: 0.36-0.66 ; P=3.10 \times 10^{-6}\right.$ for rs12007907 and $\mathrm{OR}=1.71,95 \% \mathrm{CI}: 1.36-2.15 ; P=4.57 \times 10^{-6}$; for rs199522937). Likewise, the rs12007907 and rs199522937 SNPs remained as being most associated with asthma symptoms when the results obtained for males and females separately were combined by the weighted Stouffer's method (Supplementary Table 1). This supports validity of the findings when one takes into account the possibility of differential effect size and direction between males and females as well $\mathrm{X}$-inactivation (that is, males coding as 0 and 2 instead 0 and 1 as assumed in the analysis).

The quantile-quantile-plot (QQ-plot) of the $P$-values illustrates that the observed significant associations were beyond those expected by chance (Figure 2). Furthermore, estimated genomic inflation factor $(\lambda)$ was $=1$, indicating that population genetic structure had negligible impact on association results. Regional plot of logistic regression in genotyped and imputed data were performed for the most associated region with asthma symptoms in X chromosome (Figure 3). Since the rs12007907 and other SNPs of IL1RAPL1 gene are very rare in nonAfrican populations, we performed the imputation using the African population (1000G.AFR, 20100804.tgz) according 1000 genomes as the reference population. The SNP rs12007907 is in high LD with other SNPs in the IL1RAPL1, suggesting that variants of this gene are most likely associated with asthma symptoms in this genomic region. We carried out an in silico analysis of the SNPs correlated $\left(r^{2} \geq 0.6\right)$ with rs12007907 to identify putative functional variants in the IL1RAPL1 region. We evaluated various functional annotations in our sets of polymorphisms, including predicted chromatin state segmentation, predicted DNAse hypersensitivity, and sequence conservation across mammals (Supplementary Table 2). This analysis revealed that rs714723 was the best candidate for a functional variant in the rs12007907 LD block. This SNP is located within a CCCTCbinding transcription factor (CTCF) sequence and can alter CTCF

Table 1 The 20 most associated SNPs in the X chromosome-wide association with asthma symptoms

\begin{tabular}{|c|c|c|c|c|c|c|c|c|c|}
\hline SNP & $H_{G V S}^{a}$ & Gene & Annotation & Minor allele & $M A F$ & OR & Cl (95\%) & & $P^{\mathrm{b}}$ \\
\hline rs12007907 & g. $29483892 C>A$ & ILIRAPLI & & $A$ & 0.25 & 0.49 & 0.37 & 0.67 & $3.33 E-06$ \\
\hline rs199522937 & g.85398157C >A & & $5.3 \mathrm{~kb} 5^{\prime}$ of $\mathrm{DACH} 2$ & $\mathrm{C}$ & 0.36 & 1.71 & 1.36 & 2.15 & $5.11 E-06$ \\
\hline rs11798204 & g.29482893G $>A$ & ILIRAPLI & & $A$ & 0.24 & 0.52 & 0.38 & 0.70 & $1.26 \mathrm{E}-05$ \\
\hline rs200054562 & g.86715043 T>A & & Intergenic & $\mathrm{T}$ & 0.31 & 1.68 & 1.33 & 2.13 & $1.38 \mathrm{E}-05$ \\
\hline rs138033925 & g. 12274046G >A & FRMPD4 & & $A$ & 0.06 & 2.29 & 1.49 & 3.52 & $1.58 \mathrm{E}-04$ \\
\hline rs5937530 & g.75569644G $>A$ & & Intergenic & $A$ & 0.14 & 1.74 & 1.30 & 2.33 & $1.76 \mathrm{E}-04$ \\
\hline rs73217711 & g.76088837 T>G & MIR325HG & & G & 0.14 & 1.74 & 1.30 & 2.33 & $1.79 E-04$ \\
\hline rs5938545 & & & Intergenic & $\mathrm{T}$ & 0.14 & 1.74 & 1.30 & 2.32 & $1.84 \mathrm{E}-04$ \\
\hline rs61343550 & g. $75951655 \mathrm{C}>\mathrm{T}$ & MIR325HG & & $\mathrm{T}$ & 0.14 & 1.73 & 1.30 & 2.32 & $1.96 \mathrm{E}-04$ \\
\hline rs5913230 & g. $79475728 \mathrm{C}>\mathrm{T}$ & & Intergenic & $\mathrm{T}$ & 0.46 & 1.54 & 1.22 & 1.93 & $2.15 E-04$ \\
\hline rs60956073 & g.76022310G $>A$ & & Intergenic & $A$ & 0.12 & 1.79 & 1.31 & 2.43 & $2.28 E-04$ \\
\hline rs12008592 & g.20676180 A>G & & Intergenic & $\mathrm{G}$ & 0.10 & 0.42 & 0.26 & 0.67 & $2.65 E-04$ \\
\hline rs6617245 & g.85844979G $>$ T & $\mathrm{DACH} 2$ & & $\mathrm{G}$ & 0.42 & 1.54 & 1.22 & 1.94 & $2.92 E-04$ \\
\hline rs4828133 & g. $85357492 A>G$ & & Intergenic & G & 0.24 & 1.58 & 1.23 & 2.02 & $3.10 E-04$ \\
\hline rs1453329 & g. $97365567 \mathrm{C}>\mathrm{T}$ & & Intergenic & $\mathrm{T}$ & 0.31 & 1.56 & 1.22 & 1.99 & $3.83 E-04$ \\
\hline rs5906181 & g.46206130G $>A$ & & Intergenic & G & 0.28 & 0.62 & 0.48 & 0.81 & $4.18 E-04$ \\
\hline rs1503783 & g. $51097378 \mathrm{C}>\mathrm{T}$ & LOC105373204 & & $\mathrm{T}$ & 0.33 & 0.62 & 0.48 & 0.81 & $4.18 E-04$ \\
\hline rs59169505 & g.29472058G > A & ILIRAPLI & & $A$ & 0.14 & 0.51 & 0.35 & 0.74 & $4.35 E-04$ \\
\hline rs5925278 & g. $152163280 C>T$ & PNMA5 & & $\mathrm{T}$ & 0.24 & 1.58 & 1.22 & 2.03 & $5.09 E-04$ \\
\hline rs6625807 & g. $70843424 C>T$ & & Intergenic & $\mathrm{T}$ & 0.21 & 0.58 & 0.43 & 0.79 & $5.09 E-04$ \\
\hline
\end{tabular}

aariation description based on hg19 human genome reference sequence.

${ }^{b} P$-value of logistic regression adjusted by sex, age and three genetic PCs. 
affinity to this genomic region. Analysis using imputed data confirmed the suggestive association of rs714723 and other variants in the rs12007907 LD block with symptoms of asthma in our population (Figure 3 and Supplementary Table 2).

We followed-up the association of rs12007907 with asthma in three independent validation cohorts for which information about this SNP were available. (Supplementary Table 3). In GALA II and Pelotas populations the rs12007907 was not associated with asthma $(\mathrm{OR}=$ 1.06, 95\% CI: $0.92-1.23 ; P=0.420$ and $\mathrm{OR}=1.26,95 \%$ CI: $0.91-1.73$; $P=0.164$, respectively). In the ProAr cohort, which was recruited in the same city and is ethnically similar to the discovery sample of this study, although no significant association was observed between rs12007907 and asthma in general analysis $(\mathrm{OR}=0.96$, 95\% CI: $0.78-$ 1.18; $P=0.707)$, the rs12007907 was negatively associated with asthma among men $(\mathrm{OR}=0.45,95 \% \mathrm{CI}: 0.21-0.95 ; P=0.038)$ but not among women $(\mathrm{OR}=1.02,95 \% \mathrm{CI}: 0.82-1.26 ; P=0.862)$. Otherwise, in the
GALA II and Pelotas populations the results remained not significant when stratifying by sex. Also in the discovery set no differences according to sex were observed for the association between rs 12007907 and asthma $\left(\mathrm{OR}=0.59,95 \% \mathrm{CI}: 0.38-0.95 ; P=2,9 \times 10^{-2}\right.$ and $\mathrm{OR}=$ 0.43, 95\% CI: $0.29-0.64 ; P=2.4 \times 10^{-5}$ among men and women, respectively).

Since Th2-type cytokine are associated with atopic asthma ${ }^{40}$ and considering that IL1 family members such as (IL33/ST2) components induce Th2 polarization, ${ }^{41}$ we analyzed the production of IL-5 and IL-13 in PWM-stimulated whole-blood cultures according to the genotypes for our top SNP (rs12007907). As can be seen in Figure 4, the homozygote for A allele had a lower production of IL-13 in comparison with homozygote for $\mathrm{C}$ allele. The geometric mean of IL-13 concentration in AA genotype was 36\% lower compared with the CC genotype $(P=0.044)$. No statistically significant differences were observed comparing the heterozygous (CA) to the CC genotype

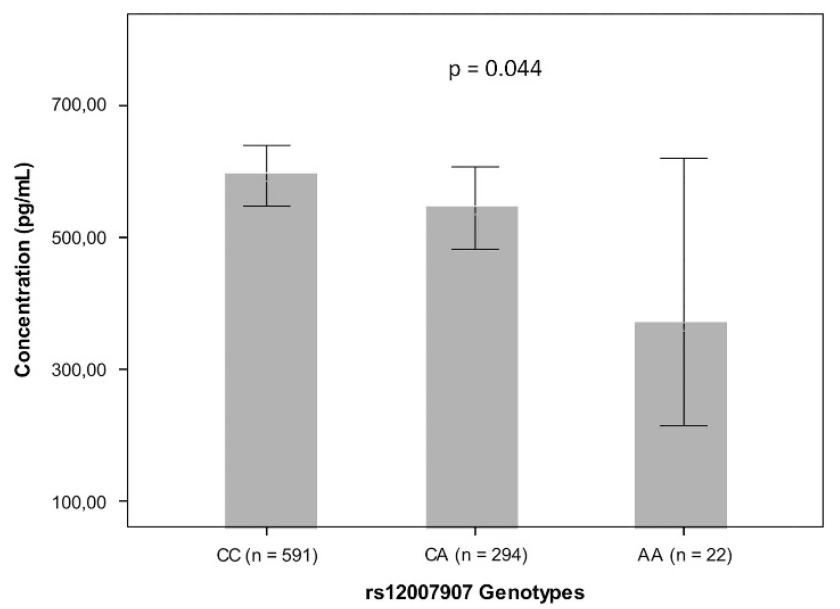

Figure 4 Geometric means for IL-13 production according to rs12007907 genotypes. The $P$-value refers to the Kruskal-Wallis test for multiple comparisons.

\section{a}

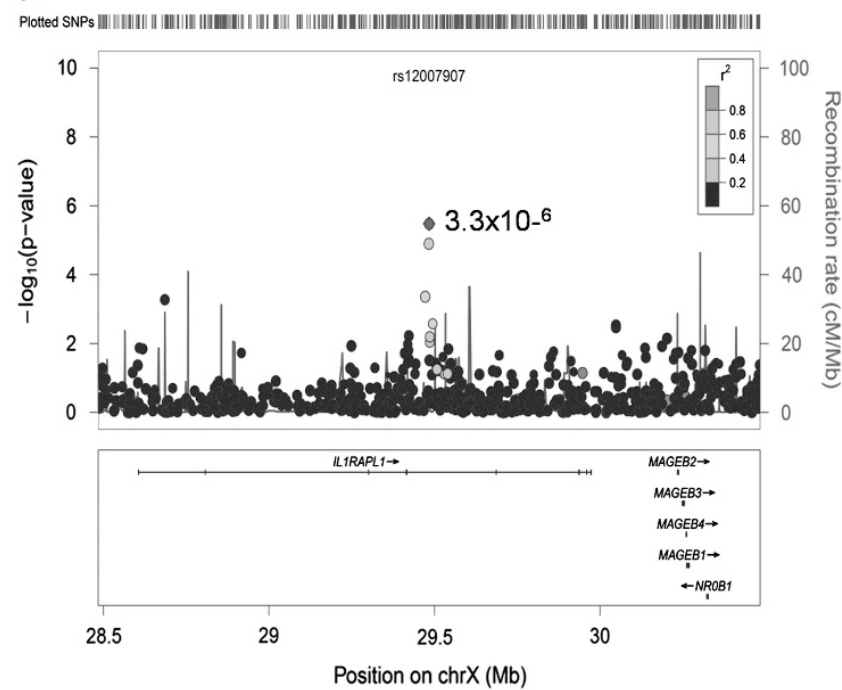

b

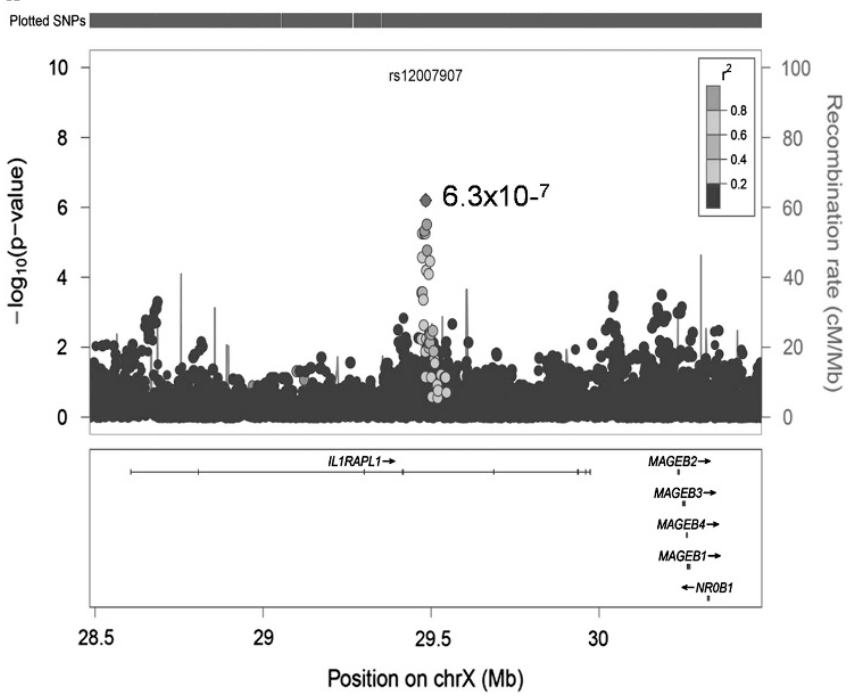

Figure 3 Regional plot for logistic regression results. The picture on the left (a) represents the results for genotyped SNPs in region most associated with asthma symptoms of $X$ chromosome. On the right (b) is represented the results for imputed SNPs in the same region. The top SNP rs12007907 is showed in purple in both $\mathbf{a}$ and $\mathbf{b}$. The full colour version of this figure is available at European Journal of Human Genetics online. 
$(P=0.278)$. The Kruskal-Wallis test also showed significant differences for IL-13 production between the different genotypic groups of rs1200797 $(P=0.044)$. No significant trend was found for IL-5 production according to rs12007907 genotype (data not shown).

The results of heterogeneity tests are shown in Supplementary Table 4. The rs56025647 was the top SNP for sex-differentiated effect size in risk for asthma on SCAALA cohort, although it did not reach a level of suggestive association considering multiple heterogeneity tests $\left(P=5.15 \times 10^{-5}\right)$. Among males, this SNP was positively associated with asthma symptoms (OR: 1.84, 95\% CI: $1.25-2.71, P=1,88 \times 10^{-3}$ ), while among females it was negatively associated (OR: 0.56, 95\% CI: 0.40 $\left.0.78, P=8,5 \times 10^{-4}\right)$. The regional plot of SNPs near to rs 56025647 is showed in Supplementary Figure 1.

The results of gene-based analysis are showed in the Supplementary Figure 2 and Supplementary Table 5. The NUDT10 was the only significantly associated gene with asthma symptoms (empirical $P$-value $<0.01)$ in both analyses based on previously computed SNP $P$-values (MAGMA and VEGAS) as well as on PCs regression (MAGMA).

The 100 SNPs most associated with asthma symptoms in the discovery set and ranked according to the $P$-value are shown in Supplementary Table 6.

\section{DISCUSSION}

The X chromosome has been often overlooked in GWAS of asthma to date. An important exception in this scenario is the Moffat et al study, ${ }^{42}$ which performed X chromosome analysis in populations with predominant European ancestry but reported no statistically significant association signals. Recently, a GWAS of asthma symptoms in Latin American children with remarkable African ancestry was carried out in the same cohort of present study, but the X chromosome was excluded from the analyses. ${ }^{43}$ However, X-WAS studies can help clarify the role of several genes in the development of many diseases, including complex illnesses such as asthma. In this context, this is to the best of our knowledge one of the first initiatives to perform an $\mathrm{X}$-WAS for asthma symptoms in Latin American children to date.

In our discovery sample two SNPs were suggestively associated with asthma symptoms. The SNP rs199522937 are intergenic and located in an upstream region of $\mathrm{DACH} 2$ gene that encodes a 599 aminoacid protein that is a transcriptional cofactor. ${ }^{42} \mathrm{DACH} 2$ sequences suggest a role in abnormalities like cleft palate and megalocornea. ${ }^{43}$ While the association of this intergenic SNP with asthma seems unclear, the top SNP in our study, located in the IL1RAPL1 gene region, suggest a possible causal effect on asthma susceptibility. The IL1RAPL1 (IL-1R accessory protein-like) is a gene that encodes a protein with 696 aminoacids homologous to IL-1 receptor accessory proteins. IL1RAPL was first identified in patients with X-linked mental retardation. ${ }^{44}$ Mutations in this gene were also identified in patients with cognitive impairment. In these cases, a loss-of-function mutation in this gene impacts on an interleukin/MAP kinase cascade of modulatory neurotransmitters affecting synapse structure leading to cognitive impairment. ${ }^{44}$ The exact function of IL1RAPL1 is not fully understood, but it is abundantly expressed in postnatal brain structures and may interact with neuronal calcium sensor, thus possibly acting in regulation of exocytosis of secretory substances. The expression of IL1RAPL protein has been detected in the brain, heart and muscle. ${ }^{45}$ Khan et al showed that IL1RAPL can active c-Jun amino-terminal kinases (JNKs) but not extracellular signal-regulated kinases (ERKs) nor mitogen-activated protein kinases (MAPKs). ${ }^{46}$ Although IL1R have high affinities for their ligands, the accessory proteins cannot do it directly. Furthermore, the function of IL1RAPL might be more related to the Toll-like receptors subfamily than IL1R subfamily. ${ }^{45}$ Interestingly, IL1RAPL1 has already been reported in two previous GWAS to be associated with cardiovascular disease ${ }^{47}$ and autism. ${ }^{19}$ Although this gene belongs to the IL1R family, it has been poorly studied, possible due to its localization in the $\mathrm{X}$ chromosome and consequent exclusion from genome-wide analysis. The rs12007907 is an intronic SNP which is in LD with others SNPs located in a regulatory region of the IL1RAPL1 gene, such as the rs714723 variant located within a CTCF sequence. Binding of target sequence elements by CTCF can block the interaction between enhancers and promoters, therefore limiting the activity of enhancers to certain functional domains. CTCF can also act as a chromatin barrier by preventing the spread of heterochromatin structures. ${ }^{48}$ The primary role of CTCF is thought to be in regulating the structure of chromatin. Therefore, SNPs located in these regions could influence gene expression. However, further analyzes including functional assays are required to identify potential causal polymorphisms located in IL1RAPL gene or even in nearby genomic regions.

Different IL-1 receptors, such as IL-1R, are found in the human genome and the IL1RAPL is a member of this subfamily. IL-1R is potential regulator of inflammation and is critical for innate immunity and host defense against infections. ${ }^{31}$ IL-1R also play an important role in the regulation of $\mathrm{TH} 2$ cell in allergic airway inflammation. ${ }^{41}$ Previous GWASs reported associations of IL1R SNPs with asthma in different populations, ${ }^{49-51}$ suggesting that IL1R gene may be involved in asthma susceptibility. Assuming that IL1RAPL gene may play a similar role of ILIR in inflammatory responses mediated by TH2 cells, we decided to evaluate the association between the rs12007907 genotypes and the production of Th2-type cytokines (IL-5 and IL-13) in our study population. Interestingly, the homozygous genotype for the rs1200797 allele negatively associated with asthma symptoms (AA genotype) was negatively associated with IL-13 production. These results suggest a possible involvement of SNPs in IL1RAPL1 gene regulating IL-13 production, which needs to be further, investigated. However, that could also explain the negative association observed for rs12007907 with asthma since IL-13 is a classical cytokine related to allergic asthma leading to bronchial hyper reactivity as well as mucus production. ${ }^{52}$

In our validation samples we did not observe significant associations in general analysis. This can be explained, at least in part, by differential frequencies of ILIRAPL1 SNPs between the populations evaluated. In fact, a pattern of biogeographical variation is observed for rs 12007907 , with allele frequencies varying from 37\% among Africans, 3\% among Europeans and 7\% among Amercan Indians. In SCAALA (the discovery sample) rs12007907 MAF (corresponding to the A allele) was $25 \%$, reflecting the remarkable African contribution to this admixed population. ${ }^{45}$ In the ProAr cohort the frequency of this SNP was 23\%, which reflects the closest genetic similarity of this population with the discovery sample. However, in the GALA II and Pelotas cohorts the MAFs were 7 and 11\%, respectively, which may result in a lack of statistical power to detect an association. In addition, in the Pelotas cohort the outcome of asthma symptoms was investigated during adolescence. ${ }^{31}$ Also, GALA II consists of adolescents and young adults, ${ }^{30}$ while the ProAr cohort is exclusively composed of adult individuals and asthma phenotype different from SCAALA (SCAALA cohort which comprises mild asthmatics only while ProAr mild and severe asthmatics). Given the heterogeneity of asthma across different stages of the life course, it is possible that genetic variants associated with childhood-onset disease are not the same implicated in late-onset disease, which is in accordance to evidence from GWAS. ${ }^{42}$ Interestingly, in the analysis stratified by sex 
the rs12007907 was negatively associated with asthma among men in ProAR cohort, in agreement with that observed in the discovery set. These results suggest that studies in admixed populations with predominantly non-European ancestry can shed light on genetic factors involved in gender differences for asthma prevalence.

Gene-based analysis revealed that NUDT10 was the most consistently associated with asthma symptoms in SCAALA-Salvador cohort. This gene belongs to NUDT gene family (NUDT = 'Nudix [Nucleoside Diphosphate attached moiety ' $x$ ']-Type motif) and encode a phosphatase involved in the diphosphoinositol polyphosphates cell turnover. ${ }^{46}$ The diphosphoinositol polyphosphates have been implicated in several cellular activities, such as homolog recombination, vesicle trafficking, apoptosis and response to environmental stress. ${ }^{47-49}$ Although association of the NUDT genes with asthma has not been previously reported, the diphosphoinositol polyphosphates syntheses is activated in cells subjected to stress by hyperosmotic pressure. ${ }^{50}$ Such osmotic stress may occur, for instance, in airways epithelial cells undergone to inadequate airway humidification caused by rapid breathing (for example, during exercise), breathing of dry/cold air and in some airway diseases. ${ }^{51}$ Interestingly, the hyperosmolality of extracellular fluid is believed to play an inflammatory role in asthma and other diseases. ${ }^{52}$ However, further studies are needed to clarify the role, if any, of NUDT10 gene in asthma risk.

For complex diseases exhibiting sexual dimorphism, such as asthma, it is supposed that X-linked variants are more likely to exhibit different effects between males and females. ${ }^{53}$ Therefore, we performed stratified analyzes in order to identify potential variants with different effect size in asthma symptoms according to sex. The top SNP in the heterogeneity tests, rs56025647, is located in an intergenic region in Xq13. The role of genetic variants in this locus to asthma risk, however, is unknown and needs to be further investigated.

An important limitation of the present study is the relatively small sample size, which can lead to lack of power, especially because SNPs generally have small effect size. Considering an OR of 1.5 and a significance level of $5.0 \times 10^{-8}$, for example, the power reached by the study is only 30.5 and $41.2 \%$ for variants with frequencies of 20 and $25 \%$, respectively. Although not calculated, the power reached by the study in analyzes stratified by sex is even lower. Nevertheless, suggestive associations were detected when correcting for multiple testing in overall sample. However, these results require further replication in more similar samples regarding their genetic background and age, which shall be complemented with functional data to understand the impact of these herein described genes in asthma.

\section{CONCLUSION}

This is the first X chromosome-wide association study for asthma carried out to date. Our findings showed that X-linked genes were suggestively associated with asthma symptoms in children, one of which belongs to IL1R's family. This latter gene was also associated with production of pro-inflammatory cytokine IL-13. Though the X chromosome is often excluded from GWAS, important differences in morbidity and severity of asthma has been reported between males and females. This prevents the assessment of the impact of sex-linked genes in asthma. Thus our study may contribute to a better understanding of genetic factors involved in asthma development and, in particular, to the observed gender differences.

\section{CONFLICT OF INTEREST}

The authors declare no conflict of interest.

\section{ACKNOWLEDGEMENTS}

This work was supported by the Department of Science and Technology (DECIT, Ministry of Health) and National Fund for Scientific and Technological Development (FNDCT, Ministry of Science and Technology), Funding of Studies and Projects (FINEP, Ministry of Science and Technology, Brazil). We thank all the patients and field workers that have participated of this work.

1 Akdis CA: Allergy and hypersensitivity: mechanisms of allergic disease. Curr Opin Immunol 2006; 18: 718-726.

2 International Union Against Tuberculosis and Lung Disease and The International Study of Asthma and Allergies in Childhood: The global asthma report 2014. Available from http://www.globalasthmareport.org (accessed March 2016).

3 Yazdanbakhsh M, Kremsner PG, van Ree R: Allergy, parasites, and the hygiene hypothesis. Science 2002; 296: 490-494.

4 Abecasis GR, Auton A, Brooks LD et al: An integrated map of genetic variation from 1,092 human genomes. Nature 2012; 491: 56-65.

5 Pearce N, Ait-Khaled N, Beasley R et al: Worldwide trends in the prevalence of asthma symptoms: phase III of the International Study of Asthma and Allergies in Childhood (ISAAC). Thorax 2007; 62: 758-766.

6 Cooper PJ, Rodrigues LC, Cruz AA, Barreto ML: Asthma in Latin America: a public heath challenge and research opportunity. Allergy 2009; 64: 5-17.

7 Woolcock AJ, Peat JK: Evidence for the increase in asthma worldwide. Ciba Found Symp 1997; 206: 122-134.

8 Upton MN, McConnachie A, McSharry C et al: Intergenerational 20 year trends in the prevalence of asthma and hay fever in adults: the midspan family study surveys of parents and offspring. BMJ 2000; 321: 88-92.

9 Wise AL, Gyi L, Manolio TA: eXclusion: toward integrating the $X$ chromosome in genome-wide association analyses. Am J Hum Genet 2013; 92: 643-647.

10 Ross MT, Grafham DV, Coffey AJ et al: The DNA sequence of the human X chromosome. Nature 2005; 434: 325-337.

11 Bushby KM: Recent advances in understanding muscular dystrophy. Arch Dis Child 1992; 67: 1310-1312.

12 Mannucci PM, Tuddenham EG: The hemophilias-from royal genes to gene therapy. N Engl J Med 2001; 344: 1773-1779.

13 Wildin RS, Freitas A: IPEX and FOXP3: clinical and research perspectives. J Autoimmun 2005; 25(Suppl): 56-62.

14 Bottema RW, Kerkhof M, Reijmerink NE et al: Gene-gene interaction in regulatory T-cell function in atopy and asthma development in childhood. J Allergy Clin Immunol 2010; 126: 338-346.

15 Du X, Poltorak A, Wei Y, Beutler B: Three novel mammalian toll-like receptors: gene structure, expression, and evolution. Eur Cytokine Netw 2000; 11: 362-371.

16 Carey MA, Card JW, Voltz JW et al: It's all about sex: gender, lung development and lung disease. Trends Endocrinol Metab 2007; 18: 308-313.

17 Postma DS: Gender differences in asthma development and progression. Gend Med 2007; 4: S133-S146

18 Chu X, Shen M, Xie F et al: An X chromosome-wide association analysis identifies variants in GPR174 as a risk factor for Graves' disease. J Med Genet 2013; 50: 479-485.

19 Chung RH, Ma D, Wang $\mathrm{K}$ et al: An X chromosome-wide association study in autism families identifies TBL1X as a novel autism spectrum disorder candidate gene in males. Mol Autism 2011; 2: 18.

20 Conde L, Foo JN, Riby J et al: X chromosome-wide association study of follicular lymphoma. Br J Haematol 2013; 162: 858-862.

21 Figueiredo CA, Alcantara-Neves NM, Veiga R et al: Spontaneous cytokine production in children according to biological characteristics and environmental exposures. Environ Health Perspect 2009; 117: 845-849.

22 Rodrigues LC, Newcombe PJ, Cunha SS et al: Early infection with Trichuris trichiura and allergen skin test reactivity in later childhood. Clin Exp Allergy 2008; 38: 1769-1777.

23 Barreto ML, Cunha SS, Alcantara-Neves N et al: Risk factors and immunological pathways for asthma and other allergic diseases in children: background and methodology of a longitudinal study in a large urban center in Northeastern Brazil (Salvador-SCAALA study). BMC Pulm Med 2006; 6: 15.

24 Figueiredo CA, Barreto ML, Rodrigues LC et al: Chronic intestinal helminth infections are associated with immune hyporesponsiveness and induction of a regulatory network. Infect Immun 2010; 78: 3160-3167.

25 Alcantara-Neves NM, Veiga RV, Dattoli VC et al: The effect of single and multiple infections on atopy and wheezing in children. J Allergy Clin Immunol 2012; 129: 359-367.

26 Figueiredo CA, Amorim LD, Alcantara-Neves NM et al: Environmental conditions, immunologic phenotypes, atopy, and asthma: new evidence of how the hygiene hypothesis operates in Latin America. J Allergy Clin Immunol 2013; 131: 1064-1068.

27 Kehdy FS, Gouveia MH, Machado $M$ et al: Origin and dynamics of admixture in Brazilians and its effect on the pattern of deleterious mutations. Proc Natl Acad Sci USA 2015; 112: 8696-8701.

28 Cooper GM, Stone EA, Asimenos G, Green ED, Batzoglou S, Sidow A: Distribution and intensity of constraint in mammalian genomic sequence. Genome Res 2005; 15: 901-913. 
29 ENCODE Project Consortium: An integrated encyclopedia of DNA elements in the human genome. Nature 2012; 489: 57-74.

30 Nishimura KK, Galanter JM, Roth LA et al: Early-life air pollution and asthma risk in minority children. The GALA II and SAGE II studies. Am J Respir Crit Care Med 2013; 188: 309-318.

31 Victora CG, Barros FC: Cohort profile: the 1982 Pelotas (Brazil) birth cohort study. Int J Epidemiol 2006; 35: 237-242.

32 Van Ganse E, Antonicelli L, Zhang Q et al: Asthma-related resource use and cost by GINA classification of severity in three European countries. Respir Med 2006; 100 140-147.

33 Figueiredo CA, Alcantara-Neves NM, Amorim LD et al: Evidence for a modulatory effect of IL-10 on both Th1 and Th2 cytokine production: the role of the environment. Clin Immunol 2011; 139: 57-64.

34 Salzano FM, Bortolini MC: The Evolution and Genetics of Latin American Populations. New York: Cambridge Univ Press, 2002.

35 Willer CJ, Li Y, Abecasis GR: METAL: fast and efficient meta-analysis of genomewide association scans. Bioinformatics 2010; 26: 2190-2191.

36 Fuchsberger C, Abecasis GR, Hinds DA: minimac2: faster genotype imputation. Bioinformatics 2014; 31: 782-784.

37 Howie B, Fuchsberger C, Stephens M, Marchini J, Abecasis GR: Fast and accurate genotype imputation in genome-wide association studies through pre-phasing. Nat Genet 2012; 44: 955-959.

38 Liu JZ, McRae AF, Nyholt DR et al: A versatile gene-based test for genome-wide association studies. Am J Hum Genet 2010; 87: 139-145.

39 de Leeuw CA, Mooij JM, Heskes T, Posthuma D: MAGMA: generalized gene-set analysis of GWAS data. PLoS Comput Biol 2015; 11: e1004219.

40 Barnes PJ: Th2 cytokines and asthma: an introduction. Respir Res 2001; 2: 64-65.

41 Dunne A, O'Neill LA: The interleukin-1 receptor/Toll-like receptor superfamily: signal transduction during inflammation and host defense. Sci STKE 2003; 2003: re3.

42 Moffatt MF, Gut IG, Demenais F et al: A large-scale, consortium-based genomewide association study of asthma. N Engl J Med 2010; 363: 1211-1221.
43 Costa GN, Dudbridge F, Fiaccone RL et al: A genome-wide association study of asthma symptoms in Latin American children. BMC Genet 2015; 16: 141.

44 Born TL, Smith DE, Garka KE, Renshaw BR, Bertles JS, Sims JE: Identification and characterization of two members of a novel class of the interleukin-1 receptor (IL-1R) family. Delineation of a new class of IL-1R-related proteins based on signaling. J Biol Chem 2000; 275: 29946-29954.

45 Lima-Costa MF, Rodrigues LC, Barreto ML et al: Genomic ancestry and ethnoracial selfclassification based on 5,871 community-dwelling Brazilians (The Epigen Initiative) Sci Rep 2015; 5: 9812.

46 Shears SB, Gokhale NA, Wang H, Zaremba A: Diphosphoinositol polyphosphates: what are the mechanisms? Adv Enzyme Regul 2011; 51: 13-25.

47 Barker CJ, Illies C, Gaboardi GC, Berggren PO: Inositol pyrophosphates: structure, enzymology and function. Cell Mol Life Sci 2009; 66: 3851-3871.

48 Burton A, Hu X, Saiardi A: Are inositol pyrophosphates signalling molecules? J Cell Physiol 2009; 220: 8-15.

49 Hua LV, Hidaka K, Pesesse X, Barnes LD, Shears SB: Paralogous murine Nudt10 and Nudt11 genes have differential expression patterns but encode identical proteins that are physiologically competent diphosphoinositol polyphosphate phosphohydrolases. Biochem J 2003; 373: 81-89.

50 Pesesse X, Choi K, Zhang T, Shears SB: Signaling by higher inositol polyphosphates. Synthesis of bisdiphosphoinositol tetrakisphosphate ('InsP8') is selectively activated by hyperosmotic stress. J Biol Chem 2004; 279: 43378-43381.

51 Song Y, Jayaraman S, Yang B, Matthay MA, Verkman AS: Role of aquaporin water channels in airway fluid transport, humidification, and surface liquid hydration. J Gen Physiol 2001; 117: 573-582.

52 Neuhofer W: Role of NFAT5 in inflammatory disorders associated with osmotic stress. Curr Genomics 2010; 11: 584-590.

53 Dobyns WB, Filauro A, Tomson BN et al: Inheritance of most X-linked traits is not dominant or recessive, just X-linked. Am J Med Genet A 2004; 129A: 136-143.

Supplementary Information accompanies this paper on European Journal of Human Genetics website (http://www.nature.com/ejhg) 